\title{
Motor Imagery and Swallowing: Introduction to Literature and Discussion of Research needs in Dysphagia
}

Szynkiewicz $\mathrm{SH}^{1 *}$, Nobriga $\mathrm{CV}^{2}$ and Donoghue $\mathrm{CRO}^{3}$

${ }^{1}$ University of South Florida, USA

${ }^{2}$ Loma Linda University, USA

${ }^{3}$ James Madison University, USA

\begin{abstract}
Background: A large base of neurophysiological and neurobehavioral research supports motor imagery as an effective high-level, cognitive therapeutic strategy to enhance physical rehabilitation. One creates an imagined mental representation of a motor task without physical execution of the task. Mental practice, the repetitive rehearsal of a motor imagery task, complements physiotherapy treatment (active exercise) and has been shown to lead to increased motor learning and motor performance outcomes.

Aim and main contribution: The current paper provides a review of dysphagia and exercise rehabilitation, an introduction to motor imagery and mental practice in clinical rehabilitation, a discussion of emerging research of motor imagery of swallowing and swallowing-related movements, and potential novel and exciting applications of motor imagery to dysphagia rehabilitation that warrant investigation.

Conclusion: Despite an extensive research base supporting the benefit of using mental practice in addition to active exercise following neurological injury, the potential applications for dysphagia remain largely under-investigated. Given the need for evidence-based, cost-effective, and accessible clinical management, this paper discusses therapeutic applications important for preventative and rehabilitative considerations for motor imagery and encourages expanded research in the area of motor imagery and dysphagia.
\end{abstract}

Keywords: Deglutition disorders; Dysphagia; Speech-language pathology; Exercise; Mental processes; Rehabilitation; Motor imagery; Mental practice

\section{Introduction}

Dysphagia, difficulty swallowing, refers to atypical swallowing physiology and/or anatomy resulting in reduced safety and/or efficiency of functional swallowing ability [1]. In such cases, the normal transport of food, liquid or saliva through the upper aerodigestive tract to the cervical esophagus is disrupted. In adults, dysphagia generally results from a primary neurological injury or progressive disease, neuromuscular disease, head and neck cancer anatomical anomaly or treatment, or pulmonary disease [1]. However, it may also result from sarcopenia (as a result of the normal aging process or secondary to sequelae resulting from disease) of swallowing musculature $[2,3]$. The prevalence of dysphagia increases when considering the sarcopenic population of older adults. Up to $40 \%$ of adults over the age of 60 experience dysphagia [4-6]. With the projected growth in number of aging adults, the incidence of dysphagia in the geriatric population is likely to increase [7]. Health-related consequences of dysphagia include pneumonia, malnutrition/dehydration, and increased mortality [1]. Significant costs each year are associated with hospitalizations involving patients with dysphagia [8].

\section{Dysphagia and the Aging Process}

A syndrome associated with the typical aging process, sarcopenia, produces a reduction in the mass and strength of skeletal muscles affecting musculature important to swallowing [2]. In primary form, sarcopenia is related to the typical aging process [9]. Sarcopenia is considered secondary when related to activity, disease, or nutritional changes [9]. The etiology of sarcopenic dysphagia can be multifactorial and either form of sarcopenia can lead to a decline in swallowing function, nutritional status, and activities of daily and independent living abilities [2]. Sarcopenic dysphagia is not to be confused with presbyphagia, which is associated with changes in the swallowing mechanism related to typical aging but do not lead to disordered functional swallowing [3]. However, even presbyphagia places older adults at a higher risk for dysphagia, perhaps representing a pre-dysphagic state, as these typical changes in the swallowing mechanism with aging include changes in swallowing anatomy, oropharyngeal timing during swallowing $[1,10]$, reductions in head and neck structural movement [11,12], decreased lingual strength and functional reserve [13] and cortical activation for swallowing [14]. While rehabilitative measures have traditionally been the focus, preventative strategies are also being recognized as critical in research, especially with the aging population given its susceptibility to developing difficulty swallowing [15].

\section{Managing Dysphagia in the Aging Population}

Speech-language pathologists (SLPs) evaluate, diagnose, and treat patients with dysphagia, with three primary goals: safety, efficiency, and quality of life [1]. Keeping a patient as safe as possible when oral intake is indicated and preventing aspiration (i.e., ingested material or saliva aberrantly traveling below the level of the true vocal folds) is a primary focus. In addition, SLPs ensure the patient is able to intake their meals efficiently to support hydration and nutrition. As eating is a significant

${ }^{*}$ Corresponding author: Szynkiewicz SH, University of South Florida, SarasotaManatee, College of Science and Mathematics, 8350 N. Tamiami Trail, Sarasota FL 34243, USA, Tel: 941-359-4383; Fax: 941-359; E-mail: sehegyi@usf.edu

Received December 27, 2017; Accepted January 15, 2018; Published January 22, 2018

Citation: Szynkiewicz SH, Nobriga CV, Donoghue CRO (2018) Motor Imagery and Swallowing: Introduction to Literature and Discussion of Research needs in Dysphagia. Health Care Current Reviews 6: 218. doi: 10.4172/2375-4273.1000218

Copyright: () 2018 Szynkiewicz SH, et al. This is an open-access article distributed under the terms of the Creative Commons Attribution License, which permits unrestricted use, distribution, and reproduction in any medium, provided the original author and source are credited. 
part of one's quality of life, this should always be incorporated during evaluation and treatment. Rehabilitative therapeutic interventions in dysphagia target direct changes to muscles and neural activation for swallowing in order to improve functional swallowing performance [1,16-18]. Active exercise, including swallowing and non-swallowing exercises have been included in rehabilitative dysphagia intervention for decades [17]. Active exercise of muscles important to swallowing in dysphagia treatment is believed to improve biomechanics of swallowing function [18]. Active exercise also fosters neuroplasticity and retrains neuromuscular systems involved in swallowing [16,17,19-22]. The field of speech-language pathology is still investigating the application of the principles of motor learning and training to bulbar musculature and functional swallowing improvement; however, a solid base of research points to the reversal of muscle strength loss with active exercise, even in older adults [18]. Promising outcomes include enhanced functional swallowing with improved protection of the airway, safer and more efficient bolus transport through the upper aerodigestive tract, nutritional improvements and higher ratings with quality of life measurement tools $[18,23]$.

\section{Motor Imagery and Clinical Rehabilitation}

Motor imagery (MI) is a mind-body, cognitive, rehabilitation tool that refers to the creation of an imagined motor task without overt motor movement as the motor task is completed in working memory [24,25]. More simply stated, it is thinking about completing an action. Neural circuitry and cortical representations are receptive to modification and reorganization through experience (disuse or retraining) [26-29]. As with physiotherapy treatment, mental practice via MI contributes to activity-dependent experience required to drive neuroplasticity in neuromuscular systems to influence patterns of movement in both healthy populations and during motor rehabilitation [28]. Research indicates that MI activates similar neural substrates as active execution, even following a neurological insult [28]. A large body of literature indicates the promise of using $\mathrm{MI}$ in physical and occupational rehabilitation to enhance active exercise in motor learning and motor performance post-neurologic injury [30,31]. MI exercise, or mental practice, is not generally considered as a replacement for active exercise, rather to supplement active exercise [32-35]. In fact, the most effective approach appears to be when physiotherapy treatment is combined with mental practice, with motor performance outcomes increased when motor MI is included [30,32-35]. An extensive and growing body of literature supports $\mathrm{MI}$ as an effective cognitive tool for physical rehabilitation [36]. Despite the promising evidence, potential applications of MI and swallowing have only begun to receive attention in the area of dysphagia rehabilitation.

\section{Motor Imagery and Swallowing}

Just as corticospinal reorganization has been demonstrated, empirical evidence supports swallowing neuroplasticity [22,37,38]. Physiotherapy treatment during dysphagia rehabilitation is used to retrain neuromuscular systems important to swallowing and drive neuroplasticity to influence behavioral, functional swallowing improvement [16,17,19-22]. However, only a small number of research papers explore the potential for motor imagery (MI) of swallowing to enhance dysphagia rehabilitation. Research targeting neural correlates of head and neck movements related to swallowing during MI and detection of MI of swallowing is emerging [39-44]. Innovative approaches to improving detection of MI of swallowing using electroencephalogram continue [45]. A research team has also investigated the neural correlates of MI during swallowing in healthy adults and adults with dysphagia using near-infrared spectroscopy (NIRS) $[46,47]$. The hemodynamic response during cortical activation shows a comparable pattern and topographical distribution when MI of swallowing is compared to motor execution of swallowing in both healthy adults and adults with dysphagia $[46,47]$. Further, the same research team has demonstrated cortical reorganization in motor areas of the cortex important to swallowing following neurofeedback training with NIRS using MI of swallowing in young adults [48]. The promise of using MI during dysphagia rehabilitation following neurological insult is recognized and is growing in focus and potential application [41,49]. The possible ability to harness comparable cortical activation systems for swallowing using motor MI is leading to the development of innovative therapeutic tools and techniques in dysphagia rehabilitation [49].

\section{Exercise Principles and MI for Swallowing}

Interestingly, a critique of current literature reviewing the efficacy of rehabilitative swallowing exercises in patients with dysphagia noted that primarily non-swallowing exercises such as Shaker Head Lift and Expiratory Muscle Strength Training were determined to have enough evidence for use with certain patient populations [17]. Tongue strengthening exercises are also a form of non-swallowing exercise that have "great potential" and warrant investigation using larger sample sizes and improved methodological study design, including samples with increased uniformity of subjects $[17,23]$. Non-swallowing exercises target important parts of swallowing but do not involve task-specific swallowing activity as part of the exercise [17]. Further, non-swallowing exercises do not meet the "use it or lose it," "use it and improve it" or "specificity" principles of motor learning for neuroplasticity $[17,22]$. Non-swallowing exercises target and capitalize on the motor principle of transference, just as strength training for an athlete contributes to increased motor performance of a sports skill $[16,17,22]$. The finding that non-swallowing exercise carries evidence for use in disordered populations may stem from the need for improved study design and larger sample sizes [17].

Principles of motor learning and neural plasticity that appear not to have received as much attention in the area of dysphagia exercise are the individual's interest in practice and practicing the correct form of a motor skill. Both are principles that may be supported by mental practice. Application of principles of motor learning and neural plasticity for smaller, bulbar musculature warrants attention. Muscles critical to deglutition, particularly the tongue muscles, are unique in muscle composition and their hybrid fiber types are different from other skeletal muscle in humans $[16,50]$. The bulbar musculature may demonstrate unique needs and responses in relation to the principles of neuromuscular training. The rules and principles of strength training would likely benefit from further exploration for oropharyngeal muscles. The challenge and the need to investigate "therapies that apply and/or manipulate different aspects of strength-training principles to optimize outcomes through the most efficacious, efficient, effective approaches possible" are recognized in dysphagia rehabilitation [16].

Furthermore, for decades, dysphagia rehabilitation has focused on the physiotherapy aspects of swallowing in rehabilitation. Growing evidence supports that central-level factors significantly contribute to motor outcomes like strength gain [51]. The cognitive and perceptual processes that continuously interact with the motor system both need to be considered in clinical rehabilitation [25]. Mental practice using motor imagery may serve as an innovative therapeutic tool for enhancing dysphagia exercise rehabilitation without inducing pain or 
Citation: Szynkiewicz SH, Nobriga CV, Donoghue CRO (2018) Motor Imagery and Swallowing: Introduction to Literature and Discussion of Research needs in Dysphagia. Health Care Current Reviews 6: 218. doi: 10.4172/2375-4273.1000218

fatigue. The mental practice may prime subsequent swallowing tasks for improved performance through similar neural activation patterns as the actual motor task. The addition of mental practice to complement active swallowing exercise may lead to the greatest increase in motor performance, as it has been shown in research in the limbs musculature. Further, the contribution of neural adaptations in strength gain may be greater in the older population than morphological adaptation $[16,52]$. The combination of mental and physical exercise may maximize adaptation at both the central and peripheral levels.

\section{Discussion and Future Directions}

Thus far, the focus in growing attention to motor imagery (MI) of swallowing and dysphagia rehabilitation has used sophisticated neurofeedback during MI training and cortical reorganization for swallowing [41,45-49]. The contribution of using MI of swallowing and swallowing-related movements to enhance motor learning and motor performance has potential application in both preventative and rehabilitative arenas. Practical, cost-effective and accessible applications are needed, as most patients and speech-language therapists do not have access to sophisticated equipment for neurofeedback and detection of MI of swallowing. Mental practice via MI is voluntarily driven and accessible beyond the traditional therapeutic setting once trained [25,53]. Mental practice can be imagined in varied environmental contexts, meaning the correct motoric form of swallowing or swallowing-related movement can be practiced in working memory under different simulated feeding and exercise conditions. Further, MI is safe and thus, may present as an attractive therapeutic tool for patients with severe dysphagia, who are often apprehensive to practice even using only their saliva [36]. Mental practice may provide an option for early dysphagia rehabilitation in the acute stage of neurological injury that is safe to rehearse as a cognitive tool to prime the neuromuscular system for later active exercise. Additionally, even though MI practice is not considered a replacement for active exercise, it may prove a useful therapeutic strategy when active exercise is not possible for a period of time, such as when active practice is too painful or fatiguing (e.g. during head and neck cancer treatment). Lastly, the potential for using MI strategies with active exercise warrants investigation for preventative means in the typically aging population at risk for dysphagia secondary to changes in swallowing musculature. The authors of this paper are exploring practical, cost-effective, and accessible applications of using MI for swallowing and swallowing-related movements to enhance the efficacy (e.g. strength, endurance) of active exercise during dysphagia rehabilitation. Mental practice using MI of swallowing and swallowingrelated movements represents an innovative and exciting therapeutic direction. Research exploring potential applications for preventative and rehabilitative measures focused on the function of oropharyngeal musculature for swallowing is promising and encouraged.

\section{References}

1. Logemann JA (1998) Evaluation and treatment of swallowing disorders. PROED, Austin.

2. Shiozu H, Higashijima M, Koga T (2015) Association of sarcopenia with swallowing problems, related to nutrition and activities of daily living of elderly individuals. J Phys Ther Sci 27: 393-396.

3. Wakabayashi H (2014) Presbyphagia and sarcopenic dysphagia: Association between aging, sarcopenia and deglutition disorders. J Frailty Aging 3: 97-103.

4. Doggett DL, Turkelson CM, Coates V (2002) Recent developments in diagnosis and intervention for aspiration and dysphagia in stroke and other neuromuscular disorders. Curr Atheroscler Rep 4: 311-318.

5. Feinberg MJ, Knebl J, Tully J, Segall L (1990) Aspiration and the elderly Dysphagia 5: 61-71.
6. Robbins J, Gangnon RE, Theis SM, Kays SA, Hewitt AL, et al. (2005) The effects of lingual exercise on swallowing in older adults. J Am Geriatr Soc 53 1483-1489.

7. Aslam M, Vaezi MF (2013) Dysphagia in the elderly. Gastroenterol Hepatol (NY) 9: 784-795

8. Altman K, Yu G, Schaefer S (2010) Consequence of dysphagia in the hospitalized patient: Impact on prognosis and hospital resources. Arch Otolaryngol Head Neck Surg 136: 784-789.

9. Cruz-Jentoft AJ, Baeyens JP, Bauer JM, Boirie Y, Cederholm T, et al. (2010) Sarcopenia: European consensus on definition and diagnosis: Report of the European Working Group on sarcopenia in older people. Age Ageing 39: 412 423.

10. Nagaya M, Sumi $Y$ (2002) Reaction time in the submental muscles of normal older people. J Am Geriatr Soc 50: 975-976.

11. Logemann JA, Pauloski BR, Rademaker AW, Colangelo LA, Kahrilas PJ, et al (2000) Temporal and biomechanical characteristics of oropharyngeal swallow in younger and older men. J Speech Lang Hear Res 43: 1264-1274.

12. Logemann JA, Pauloski BR, Rademaker AW, Kahrilas PJ (2002) Oropharyngea swallow in younger and older women: Video fluoroscopic analysis. J Speech Lang Hear Res 45: 434-445.

13. Nicosia MA, Hind JA, Roecker EB, Carnes M, Doyle J, et al. (2000) Age effects on the temporal evolution of isometric and swallowing pressure. J Gerontol A Biol Sci Med Sci 55: M634-640.

14. Malandraki GA, Perlman AL, Karampinos DC, Sutton BP (2011) Reduced somatosensory activations in swallowing with age. Hum Brain Mapp 32: 730 743

15. Jadcherla SR, McCullough GH, Daniels SK, Van Daele DJ, Dua K, et al. (2017) The Dysphagia Research Society accelerating a priority research agenda. Dysphagia 32: 11-14

16. Burkhead LM, Sapienza CM, Rosenbek J (2007) Strength-training exercise in dysphagia rehabilitation: Principals, procedures and directions for future research. Dysphagia 22: 251-265

17. Langmore SE, Pisegna JM (2015) Efficacy of exercises to rehabilitate dysphagia: A critique of the literature. Int J Speech Lang Pathol 17: 222-229.

18. Ney DM, Weiss JM, Kind AJ, Robbins J (2009) Senescent swallowing: Impact, strategies and interventions. Nutr Clin Pract 24: 395-413.

19. Clark HM (2003) Neuromuscular treatments for speech and swallowing: A tutorial. Am J Speech Lang Pathol 12: 400-415.

20. Fox CM, Ramig LO, Ciucci MR, Sapir S, McFarland DH, et al. (2006) The science and practice of LSVT/LOUD: Neural plasticity-principled approach to treating individuals with Parkinson disease and other neurological disorders. Semin Speech Lang 27: 283-299.

21. Kleim JA, Jones TA (2008) Principles of experience dependent neural plasticity: Implications for rehabilitation after brain damage. J Speech Lang Hear Res 51 : S225-S239.

22. Robbins J, Butler SG, Daniels SK, Gross RD, Langmore S, et al. (2008) Swallowing and dysphagia rehabilitation: Translating principles of neura plasticity into clinically oriented evidence. J Speech Lang Hear Res 51 : S276-S300.

23. McKenna VS, Zhang B, Haines M B, Kelchner LN (2017) A systematic review of isometric lingual strength-training programs in adults with and without dysphagia. Am J Speech Lang Pathol 26: 524-539.

24. Jeannerod M, Decety J (1995) Mental motor imagery: A window into the representational stages of action. Curr Opin Neurobiol 5: 727-732.

25. Mulder T (2007) Motor imagery and action observation: Cognitive tools fo rehabilitation. J Neural Transm 114: 1265-1278.

26. Buonomano DV, Merzenich MM (1998) Cortical plasticity: From synapses to maps. Annu Rev Neurosci 21: 149-186.

27. Rossini PM, Calautti C, Pauri F, Baron JC (2003) Post-stroke plastic reorganisation in the adult brain. Lancet Neurol 2: 493-502.

28. Ruffino C, Papaxanthis C, Lebon F (2017) Neural plasticity during motor learning with motor imagery practice: Review and perspectives. Neuroscience 341: 61-78. 
Citation: Szynkiewicz SH, Nobriga CV, Donoghue CRO (2018) Motor Imagery and Swallowing: Introduction to Literature and Discussion of Research needs in Dysphagia. Health Care Current Reviews 6: 218. doi: 10.4172/2375-4273.1000218

29. Sanes JN, Suner S, Lando JF, Donoghue JP (1988) Rapid reorganization of adult rat motor cortex somatic representation patters after motor nerve injury. Proc Natl Acad Sci USA 85: 2003-2007.

30. Dickstein R, Deutsch JE (2007) Motor imagery in physical therapist practice. Phys Ther 87: 942-953.

31. Peters HT, Page SJ (2015) Integrating mental practice with task-specific training and behavioral supports in post-stroke rehabilitation: Evidence, components and augmentative opportunities. Phys Med Rehabil Clin N Am 26: 715-727.

32. Driskell JE, Copper C, Moran A (1994) Does mental practice enhance performance? J Appl Psychol 79: 481-492.

33. Feltz DL, Landers DM (1983) The effects of mental practice on motor skill learning and performance: A meta-analysis. J Sport Psychol 5: 25-57.

34. Lebon F, Collet C, Guillot A (2010) Benefits of motor imagery training on muscle strength. J Strength Cond Res 24: 1680-1687.

35. Page SJ, Harnish S (2012) Thinking about better speech: Mental practice for stroke-induced motor speech impairments. Aphasiology 26: 127-142.

36. García Carrasco D, Aboitiz Cantalapiedra J (2016) Effectiveness of motor imagery or mental practice in functional recovery after stroke: A systematic review. Neurologia 31: 43-52.

37. Malandraki GA, Johnson S, Robbins J (2011) Functional MRI of swallowing: from neurophysiology to neuroplasticity. Head Neck 1: S14-20.

38. Martin RE (2009) Neuroplasticity and swallowing. Dysphagia 24: 218-229.

39. Ang KK, Chin ZY, Wang C, Guan C, Zhang H (2012) Filter bank common spatial pattern algorithm on $\mathrm{BCl}$ competition IV datasets $2 \mathrm{a}$ and $2 \mathrm{~b}$. Front Neurosci 6: 39.

40. Ge S, Wang R, Yu D (2014) Classification of four-class motor imagery employing single-channel electroencephalography. PLoS One 9: e98019.

41. JestroviÄ I, Coyle JL, SejdiÄ E (2015) Decoding human swallowing via electroencephalography: A state-of-the-art review. J Neural Eng 12: 051001.

42. Naeem M, Brunner C, Leeb R, Graimann B, Pfurtscheller G (2006) Seperability of four-class motor imagery data using independent components analysis. J Neural Eng 3: 208.
43. Morash V, Bai O, Furlani S, Lin P, Hallett M (2008) Classifying EEG signals preceding right hand, left hand, tongue and right foot movements and motor imageries. Clin Neurophysiol 119: 2570-2578.

44. Stippich C, Ochmann H, Sartor K (2002) Somatotopic mapping of the primary sensorimotor cortex during motor imagery and motor execution by functional magnetic resonance imaging. Neurosci Lett 331: 50-54.

45. Yang H, Guan C, Chua KSG, Wang CC, Soon PK, et al. (2014) Detection of motor imagery of swallow EEG signals based on the dual-tree complex wavelet transform and adaptive model selection. J Neural Eng 11: 035016.

46. Kober SE, Bauernfeind G, Woller C, Sampl M, Grieshofer P, et al. (2015) Hemodynamic signal changes accompanying execution and imagery of swallowing in patients with dysphagia: A multiple single-case near-infrared spectroscopy study. Front Neurol 6: 151.

47. Kober SE, Wood G (2014) Changes in hemodynamic signals accompanying motor imagery and motor execution of swallowing: A near-infrared spectroscopy study. Neuroimage 93: 1-10.

48. Kober SE, Gressenberger B, Kurzmann J, Neuper C, Wood G (2015) Voluntary modulation of hemodynamic responses in swallowing related motor areas: A near-infrared spectroscopy-based neurofeedback study. PLoS One 10 e0143314.

49. Yang $H$, Ang KK, Wang C, Phua KS, Guan C (2016) Neural and cortical analysis of swallowing and detection of motor imagery of swallow for dysphagia rehabilitation - A review. Prog Brain Res 228: 185-219.

50. Kent RD (2004) The uniqueness of speech among motor systems. Clin Linguist Phon 18: 495-505.

51. Clark BC, Mahato NK, Nakazawa M, Law TD, Thomas JS (2014) The power of the mind: The cortex as a critical determinant of muscle strength/weakness. J Neurophysiol 112: 3219-3226.

52. Hakkinen K, Kallinen M, Izquierdo M, Jokelainen K, Lassila H, et al. (1998) Changes in agonist-antagonist EMG, muscle CSA and force during strength training in middle-aged and older people. J Appl Physiol 84: 1341-1349.

53. Sharma N, Pomeroy VM, Baron JC (2006) Motor imagery: A backdoor to the motor system after stroke? Stroke 37: 1941-1952. 